\title{
Gamificação Aplicada à Educação: Um Mapeamento Sistemático
}

\author{
Simone de S. Borges ${ }^{1}$, Helena M. Reis ${ }^{1}$, Vinicius H. S. Durelli ${ }^{1}$, Ig I. Bittencourt ${ }^{2}$ \\ Patricia A. Jaques ${ }^{3}$ e Seiji Isotani ${ }^{1}$ \\ ${ }^{1}$ Instituto de Ciências Matemáticas e de Computação \\ Universidade de São Paulo (USP) \\ Caixa Postal 668 - 13560-970 - São Carlos - SP - Brasil \\ ${ }^{2}$ Instituto de Computação \\ Universidade Federal de Alagoas (UFAL) \\ ${ }^{3}$ Programa Interdisciplinar de Pós-Graduação em Computação Aplicada \\ Universidade do Vale do Rio dos Sinos (UNISINOS) \\ \{sborges, helenamcd, durelli, sisotani\}@icmc.usp.br, \\ pjaques@unisinos.br, ig.iberteic.ufal.br
}

Abstract. There is a growing interest in gamification as well as its applications and implications in the field of Education. Despite this increasing interest, there are no studies on the types of research being published in the area or studies covering what are the most investigated topics. As a first step towards bridging this gap, we carried out a systematic mapping to synthesize an overview of the area. Three hundred and fifty-seven studies related to gamification were analyzed. Among them 48 were related to education and only 26 met the criteria for inclusion and exclusion of articles defined in this work. These 26 studies were selected and categorized according to their contribution. As a result, a chart of the area was developed and the most investigated topics were identified indicating that most studies focus on investigating how gamification can be used to motivate students, improve their skills, and maximize learning.

Resumo. Existe um crescente interesse pela gamificação, suas aplicações e implicações. A despeito deste interesse ainda não existem estudos sobre os tipos de pesquisas que vem sendo conduzidos na área e quais os tópicos mais investigados. Assim, um mapeamento sistemático foi conduzido a fim de colaborar com uma visão geral da área. Inicialmente, 357 estudos sobre gamificação foram analisados. Dentre estes, 48 estavam relacionados com educação e apenas 26 satisfaziam os critérios de inclusão e exclusão de artigos definidos neste trabalho. Estes 26 artigos foram selecionados $e$ classificados de acordo com sua contribuição. Como resultado um mapa das pesquisas na área foi desenvolvido e os tópicos mais (e menos) investigados foram identificados apontando que a maioria dos estudos concentra-se em investigar como a gamificação pode ser utilizada na área de ensino para motivar estudantes, aprimorar suas habilidades e maximizar o aprendizado. 


\section{Introdução}

O termo "gamificação" (do original inglês: gamification) significa a aplicação de elementos utilizados no desenvolvimento de jogos eletrônicos, tais como estética, mecânica e dinâmica, em outros contextos não relacionados a jogos (Kapp, 2012). Motivados pelo crescente número de pesquisas sobre gamificação em outras áreas, o interesse pela gamificação e seu emprego têm aumentado também na área de educação (Lee e Doh, 2012; Domínguez et al., 2013). Este crescente interesse pode ser explicado, principalmente, pelo potencial da gamificação para influenciar, engajar e motivar pessoas (Kapp, 2012).

Estudos sobre o uso de jogos e elementos de jogos em educação vêm sendo realizados há algumas décadas (Malone e Lepper, 1987; Lepper e Cordova, 1996; Gee, 2003; Shaffer, 2006; Klopfer, 2008), porém nos últimos anos o interesse pelo tema vem aumentando em ritmo acelerado (Kapp, 2012). A despeito deste interesse, os autores não tem conhecimento da existência de estudos que apresentem uma visão geral sobre gamificação investigados no contexto educacional. Com o intuito de preencher tal lacuna, foi realizado um mapeamento sistemático das pesquisas existentes envolvendo gamificação e educação. O mapeamento sistemático é uma metodologia que envolve a busca por literatura a fim de verificar a natureza, a extensão e a quantidade de estudos publicados (chamados de estudos primários) na área de interesse (Petersen et al., 2008).

Neste trabalho são apresentados os resultados de um mapeamento sistemático, os elementos essenciais do protocolo de pesquisa elaborado e a forma como o processo foi conduzido. O restante deste trabalho está organizado da seguinte forma: na Seção 2 são feita as considerações iniciais sobre os objetivos do mapeamento elaborado. A Seção 3 descreve os principais elementos do protocolo de pesquisa e também como o mapeamento foi realizado. A Seção 4 apresenta os resultados do estudo. A Seção 5 discute eventuais ameaças à validade. E, finalmente, as conclusões são apresentadas na Seção 6.

\section{Considerações Iniciais}

O objetivo principal deste mapeamento é fornecer uma visão geral de pesquisas que vem sendo conduzidas sobre gamificação aplicada à área de educação. Portanto, embora existam artigos que investigam o uso de design gamificado, jogos sérios (serious games) e jogos digitais em contextos educacionais (game-based learning), eles não foram incluídos neste estudo. Além disto, é importante ressaltar que este estudo de mapeamento é parte de um projeto em andamento onde os autores investigam o uso de gamificação em ambientes de aprendizagem colaborativa apoiada por computador (Computer Supported Collaborative Learning - CSCL). Assim, como objetivo secundário deste mapeamento sistemático, pretende-se também identificar a existência de iniciativas que utilizam gamificação em ambientes de CSCL.

\section{O Processo do Mapeamento Sistemático}

O mapeamento conduzido foi baseado no processo descrito por Petersen et al. (2008), segundo o qual, há cinco passos essenciais a serem seguidos: (i) definição de questões de pesquisa, (ii) realização da pesquisa de estudos primários relevantes, (iii) triagem dos documentos, (iv) keywording dos resumos, e (v) a extração de dados e mapeamento. 
Considerando que as questões de pesquisa devem exemplificar os objetivos do estudo de mapeamento, as seguintes questões foram elaboradas:

$\mathbf{Q P}_{\mathbf{1}}$ : Em quais contextos e níveis educacionais gamificação tem sido mais investigada?

$\mathbf{Q P}_{2}$ : Quais os tipos de estudos mais investigados na área de educação e gamificação?

$\mathbf{Q P}_{3}$ : Quais são as principais técnicas de gamificação investigadas no domínio da aprendizagem colaborativa apoiada por computador?

Inicialmente foram realizados alguns testes com palavras que seriam utilizadas como palavras-chave nas buscas. Os testes consistiram na combinação da palavra gamification e de sinônimos e palavras relacionadas à educação e aprendizagem, todas no idioma inglês. Devido ao número reduzido de artigos retornados, ficou decidido que somente a palavra gamification seria utilizada como palavra-chave e que um critério de exclusão seria criado para eliminar estudos não relacionados à área de educação. Esta decisão permitiu que um número maior de artigos fossem analisados, minimizando as chances de que artigos relevantes fossem eventualmente ignorados. Portanto as buscas foram realizadas utilizando-se somente a palavra inglesa "gamification" como palavrachave e inicialmente 357 artigos foram retornados. Acredita-se que o número reduzido de artigos retornados pode ser explicado por ser a gamificação um campo de pesquisa ainda recente. As buscas foram realizadas utilizando-se as bases de dados eletrônicas apresentadas na Tabela 1. É importante ressaltar que as buscas foram conduzidas durante o mês de março de 2013, e que nenhum filtro de busca foi utilizado, como por exemplo, data da publicação.

Tabela 1. Estudos retornados por cada base de dados eletrônica e seleção final.

\begin{tabular}{|l|r|}
\hline \multicolumn{1}{|c|}{ Base de Dados } & \multicolumn{2}{c|}{ Quantidade } \\
\hline ACM Digital Library & 144 \\
\hline Elsevier (Science Direct) & 32 \\
\hline IEEE Xplore & 31 \\
\hline Scopus & 95 \\
\hline Springer & 55 \\
\hline Total & 357 \\
\hline Candidatos & 48 \\
\hline Seleção final & $\mathbf{2 6}$ \\
\hline
\end{tabular}

Na primeira etapa, todos os estudos primários recuperados foram avaliados a fim de se identificar aqueles relevantes para responder às questões de pesquisa. Após a leitura dos títulos, resumos e palavras chaves, este conjunto inicial foi reduzido para 48 artigos que continham estudos relacionados à educação. Durante esta triagem, foram aplicados critérios de inclusão e exclusão para cada estudo recuperado. Os critérios de inclusão elaborados foram os seguintes: 
- Se vários artigos relatam o mesmo estudo, apenas o artigo mais recente foi selecionado;

- Se o artigo descreve mais de um estudo, cada estudo foi avaliado individualmente.

E os seguintes critérios de exclusão:

- Artigos que não apresentam estudos referentes à educação;

- Artigos em outros idiomas que não o idioma inglês;

- Relatórios técnicos, documentos que estão disponíveis na forma de resumos ou apresentações (gray literature) e estudos secundários (ou seja, revisões sistemáticas da literatura e mapeamentos de estudos).

Em uma segunda etapa, procedeu-se à leitura dos resumos, introdução e conclusão e novamente à aplicação dos critérios de inclusão e exclusão dos 48 artigos candidatos e o resultado foi um subconjunto de 26 estudos primários ${ }^{*}$. É importante ressaltar que, em alguns casos, a leitura de outras partes dos artigos foi realizada para se proceder à seleção.

$\mathrm{Na}$ terceira etapa, os 26 estudos que compõe a seleção final foram lidos na íntegra. Foi realizada uma busca por palavras chaves e conceitos e posteriormente a combinação destas palavras chaves e conceitos permitiu a criação das categorias que serão apresentadas a seguir. Cada estudo primário foi classificado de acordo com o objetivo principal do estudo em relação à gamificação e sua aplicação na área de educação.

É necessário destacar que de acordo com os objetivos dos estudos, estas categorias podem se sobrepor, o que significa que os estudos podem ser classificados em uma ou mais categorias. As categorias identificadas foram:

Aprimorar Habilidades: Estudos que propõe o emprego de sistemas gamificados como recurso para melhorar a capacidade dos estudantes em executar atividades, usualmente, consideradas complexas e/ou repetitivas;

Desafios: Esta categoria inclui estudos cujos autores afirmam que sistemas gamificados que implementam atividades desafiadoras podem contribuir para a melhoria da aprendizagem;

Guidelines: Estudos cujos autores argumentam sobre os prós e contras da gamificação mas que no entretanto não apresentaram evidências empíricas;

Engajamento: Os estudos classificados nesta categoria apresentam recursos, abordagens e estratégias de gamificação para obter e reter a atenção dos estudantes;

Maximização do Aprendizado: Esta categoria abrange estudos que propõem soluções gamificadas para aprimorar a maneira como estudantes podem aprender, visando maximizar os resultados do processo de aprendizagem;

Mudança de Comportamento: Nesta categoria foram incluídos os estudos que visam promover algum tipo de mudança comportamental nos estudantes;

Socialização: Estudos nesta categoria discutem que a aprendizagem pode ocorrer em condições mais favoráveis quando apoiados por ferramentas sociais gamificadas.

\footnotetext{
* A relação final de estudos selecionados pode ser conferida em: http://goo.g1/KUH1m
} 


\section{Análise}

O objetivo desta seção é apresentar os detalhes do estudo realizado e as informações coletadas durante o processo de classificação destes estudos. São apresentados diversos gráficos para organizar estas informações e fornecer subsídios para responder às questões de pesquisa elaboradas.

Observando a Figura 1 (a) é possível perceber que a maioria dos estudos são voltados para o ensino superior (46\%), o que responde a questão de pesquisa $\mathbf{Q P}_{\mathbf{1}}$. Em contraste apenas dois estudos (8\%) tem como alvo o ensino fundamental. Seis dos estudos analisados (23\%) sugerem ou discutem modelos e propostas educacionais de maneira geral, sem entretanto determinar o nível educacional apropriado para as propostas. Estes estudos limitam-se a discutir possíveis benefícios e efeitos colaterais em consequência do emprego de gamificação com propósitos educacionais. Os demais estudos, discutem ou propõe a utilização de modelos gamificados para temas diversos como o ensino de idiomas (8\%), extensão universitária (1\%) ou modelos e propostas de treinamentos e tutoriais (12\%). Nenhum dos estudos selecionados apresentou pesquisas voltadas para a pré-escola, para o ensino médio ou para estudantes portadores de necessidades especiais.

As pesquisas sobre gamificação em ambientes de elearning (e.g. ambientes de educação a distância e sistemas Web de apoio a aprendizagem) são recentes. Dos 26 artigos analisados o mais antigo foi publicado em 2011. Observou-se um aumento significativo nos últimos anos relacionado as publicações. Na Figura 1 (b) é possível observar que em 2012 o número de estudos publicados foi mais que o dobro que em 2011. E considerando somente os meses de janeiro e fevereiro de 2013, quando esta pesquisa foi conduzida, três estudos já foram publicados.

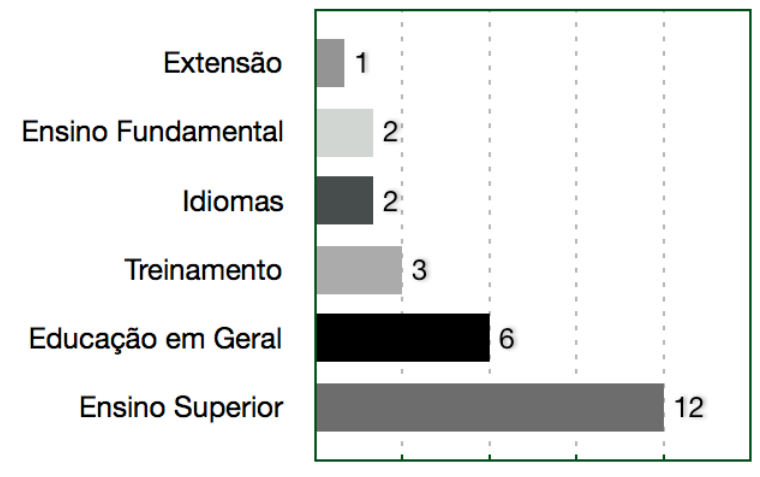

(a)

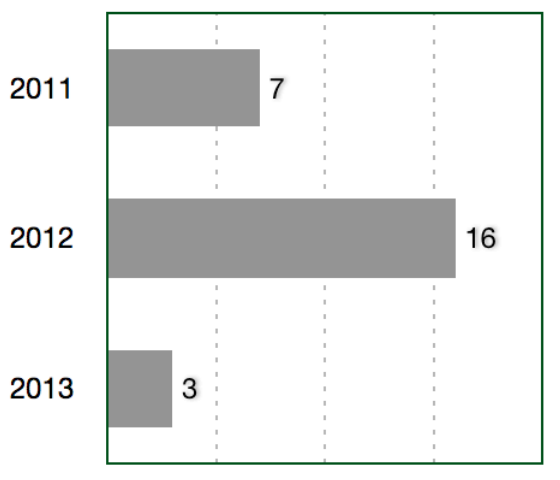

(b)

Figura 1. (a) Estudos classificados de acordo com o nível/tipo de atividade educacional e (b) pelo ano de publicação.

A maioria dos estudos analisados estavam disponíveis nas bibliotecas digitais da ACM (ACM Digital Library) e Springer como pode ser observado na Figura 2. As bibliotecas digitais da IEEE (IEEE Xplore) e Elsevier (ScienceDirect) tiveram 3 e 5 estudos selecionados respectivamente. A base de dados eletrônica Scopus também foi consultada, todavia como foi a última base a ser consultada, a maioria dos estudos relevantes já haviam sido selecionados nas bases anteriores. A consulta à Scopus resultou em quatro estudos que não foram retornados em nenhuma das bases previamente consultadas; além disso, todos foram publicados por editoras distintas. 
Baseado nas informações obtidas foi possível observar que ainda não existe um fórum dedicado ao tema, sendo que as publicações ocorreram em fóruns variados, exceto pelos comentados a seguir. Dos estudos selecionados, dois foram publicados na American Society for Engineering Education Annual Conference - ASEE 2012, dois outros foram publicados no periódico ACM eLearn Magazine, enquanto outros três estudos são capítulos que formam o livro Serious Games and Edutainment Applications (Ma et al., 2011). Todos os demais estudos foram publicados em diferentes fóruns.

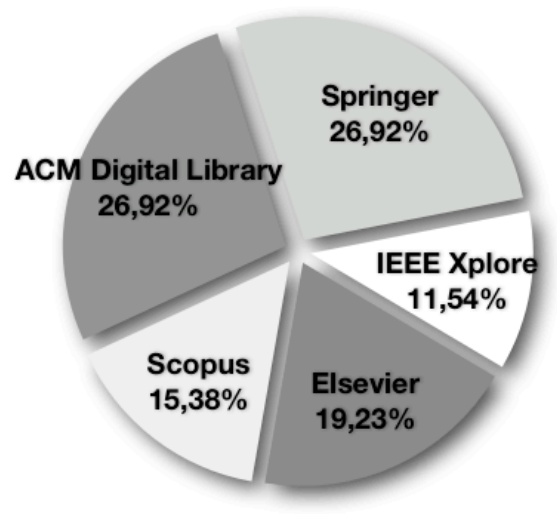

\begin{tabular}{|l|c|}
\hline \multicolumn{1}{|c|}{ Base de Dados } & Qtd. \\
\hline ACM Digital Library & $\mathbf{7}$ \\
\hline Springer & $\mathbf{7}$ \\
\hline Elsevier & $\mathbf{5}$ \\
\hline Scopus & $\mathbf{4}$ \\
\hline IEEE Xplore & $\mathbf{3}$ \\
\hline
\end{tabular}

Figura 2. Distribuição dos estudos primários de acordo com as bases consultadas.

Foram analisados estudos primários publicados em conferências, workshops, periódicos, livros, revistas e simpósios. A Figura 3 apresenta a quantidade de estudos primários selecionados de acordo com o tipo de publicação. A maioria do estudos selecionados foram publicados em conferências. Em segundo lugar ficaram os periódicos com um total de sete publicações. É importante mencionar que as áreas de interesse dos periódicos observados, em sua maioria, apresentam forte relação com diversas subáreas da ciência da computação e com educação, mas também com ciências sociais, psicologia e saúde.

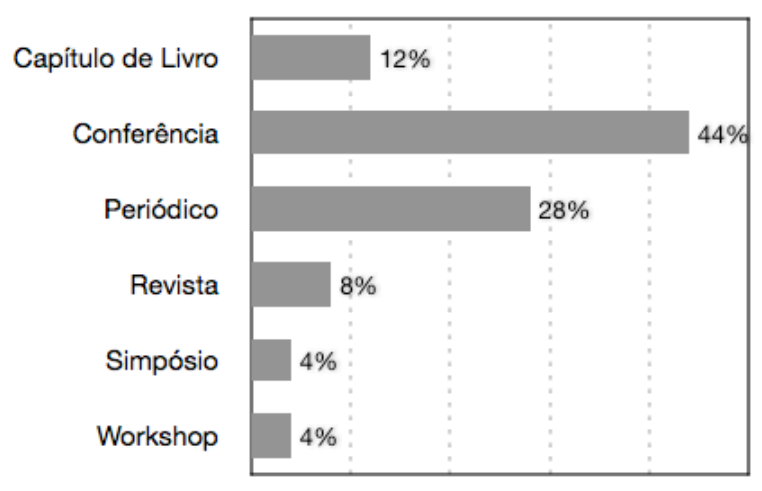

\begin{tabular}{|l|c|}
\hline \multicolumn{1}{|c|}{ Tipo de Publicação } & Qtd. \\
\hline Capítulo de Livro & 3 \\
\hline Conferência & 11 \\
\hline Periódico & 7 \\
\hline Revista & 2 \\
\hline Simpósio & 1 \\
\hline Workshop & 1 \\
\hline Total & 26 \\
\hline
\end{tabular}

Figura 3. Quantidade de estudos primários organizados por tipo de publicação.

Em todos os estudos analisados o termo "motivação" é utilizado com frequência para justificar as pesquisas desenvolvidas ou o motivo pelo qual o potencial da gamificação deve ser investigado. Percebe-se assim que a busca por inovações capazes de promover a motivação dos estudantes é uma constante em todos os estudos analisados. Entretanto, após a análise mais detalhadas das justificativas destes estudos percebeu-se que sob o termo "motivação" estão inclusos diferentes objetivos. 
Após a leitura dos estudos selecionados, foi possível identificar sete objetivos principais que foram enfatizados pelos autores: (1) aprimorar determinadas habilidades; (2) propor desafios que dão propósito/contexto a aprendizagem; (3) engajar os alunos em atividades mais participativas, interativas e interessantes; (4) maximizar o aprendizado de um determinado conteúdo; (5) promover a mudança de comportamento premiando ações adequadas e penalizando as inadequadas; (6) oferecer mecanismos de socialização e aprendizagem em grupo; e, finalmente, (7) discutir os benefícios da gamificação na motivação dos alunos para propor soluções aos diversos problemas de aprendizagem.

Além dos objetivos discutidos em cada artigo, também é importante caracterizar os tipos de estudos realizados pelos trabalhos analisados neste mapeamento. Para este fim, foi aplicada a classificação proposta por Petersen et al. (2008). Tal classificação inclui os seguintes tipos de pesquisa. Validation Research: trabalhos que apresentam uma nova técnica implementada e validada em laboratório. Evaluation Research: trabalhos que fazem uma avaliação de uma técnica implementada na prática (situação real). Opinion Papers: Estudos desse tipo relatam pontos de vista dos autores, entretanto não contém evidências que suportem essas opiniões. Philosophical Papers: são similares aos Opinion Papers, porém apresentam novas maneiras através das quais abordagens educacionais podem se beneficiar da gamificação. Solution Proposal: estudos que descrevem uma solução técnica, abordagem ou estratégia e defendem a sua utilidade, tal solução é nova ou estende uma abordagem existente; estudos nesta categoria geralmente apresentam exemplos e sólida linha de argumentação (mas não dados empíricos). Experience Papers: apresentam as experiências pessoais dos autores de um trabalho durante a inclusão de algo (e.g. uma técnica) na prática.

Com base nos tipos de estudos e nos sete objetivos identificados criou-se a Figura 4 que faz uma síntese do mapeamento realizado. A Figura 4 apresenta um mapa (do tipo bubble chart) que fornece uma visão mais precisa dos estudos realizados na área de gamificação e educação. Os estudos foram organizados da seguinte forma: no eixo $\boldsymbol{y}$ estão os sete objetivos identificados. No eixo $\boldsymbol{x}$ estão os tipos de estudos ${ }^{\dagger}$ definidos por Petersen et al. (2008).

Cada artigo analisado foi classificado como pertencendo a somente um tipo de estudo (eixo $x$ ), porém quanto aos objetivos (eixo $y$ ), os estudos poderiam ter mais de um; ou seja, os objetivos não são mutuamente exclusivos. Os valores que aparecem nas interseções entre os eixos $x$ e $y$ representam o número de estudos que citam determinado objetivo e pertencem a um determinado tipo de estudo. O tamanho de cada circunferência (bubble) é determinado pelo número de estudos classificados em ambos os pares de categorias.

Observando o mapa da Figura 4 é possível verificar que a maior parte dos estudos selecionados tem como objetivo principal avaliar (evaluation) o engajamento dos estudantes por meio da gamificação (13 estudos), enquanto que nenhum estudo dos tipos Experience ou Validation foram localizados o que responde a questão $\mathbf{Q} \mathbf{P}_{2}$.

\footnotetext{
${ }^{\dagger}$ Manteve-se os nomes dos tipos de estudos em Inglês para não alterar o sentido proposto pelo autor.
} 


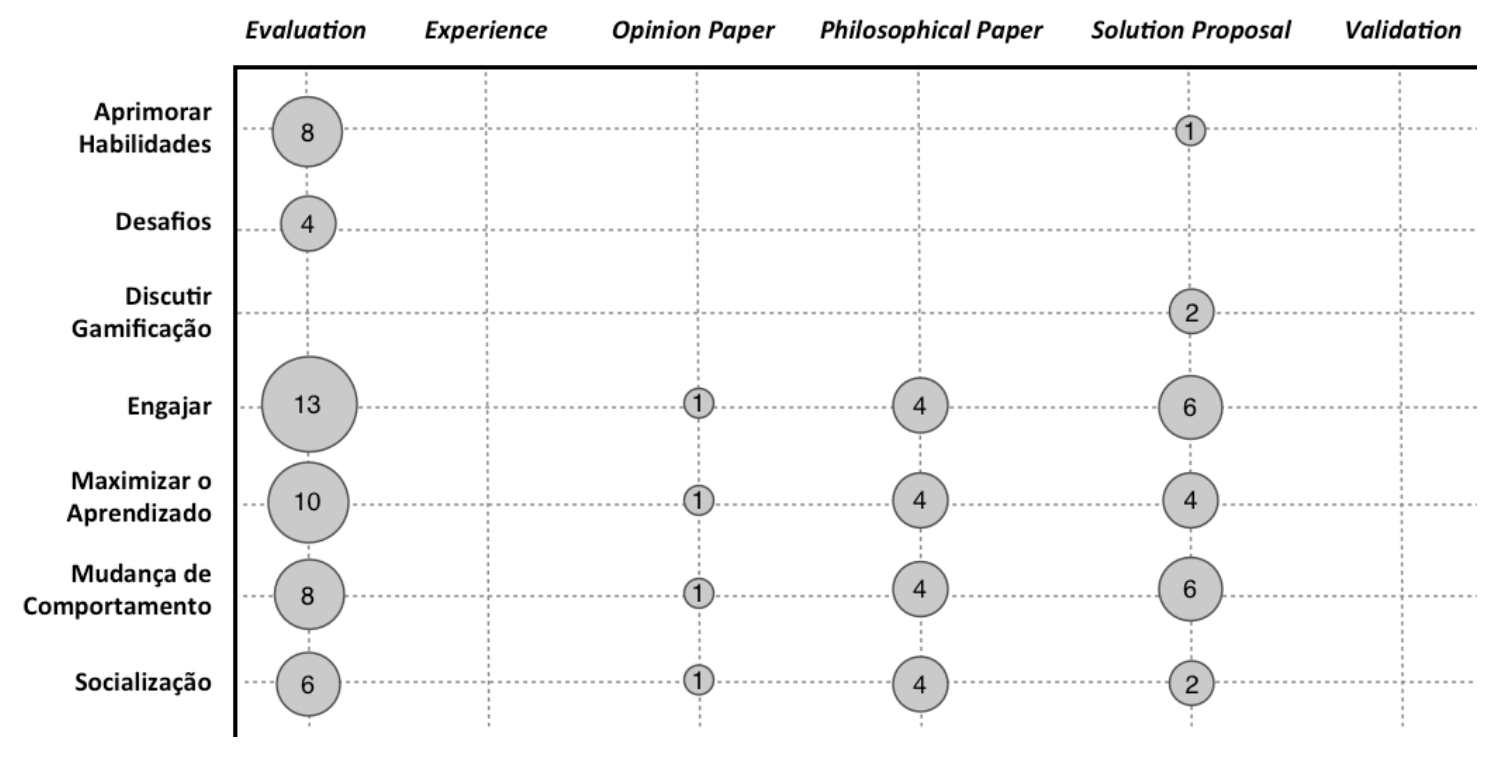

Figura 4. Mapa da distribuição dos estudos por tipo de estudo (eixo x) e objetivos da pesquisa (eixo y).

A Tabela 2 sintetiza a quantidade de estudos primários classificados por cada objetivo identificado. Promover o engajamento dos estudantes é citado por 24 dos 26 estudos selecionados. Apenas 2 estudos, ambos do tipo opinion paper, não abordam diretamente o tema, discutindo a motivação em um nível mais alto de abstração.

Também é possível observar que treze estudos citam entre seus objetivos a socialização dos estudantes como ferramenta para promover a motivação e melhorar o nível do aprendizado, entretanto somente sete apresentam soluções concretas para que isto ocorra, dois incentivam a realização de atividades em grupo, enquanto um comenta a importância deste tipo de atividade.

Tabela 2. Quantidade de estudos primários em cada categoria.

\begin{tabular}{|l|c|}
\hline \multicolumn{1}{|c|}{ Objetivo } & Qtd. \\
\hline Aprimorar Habilidades & 9 \\
\hline Desafios & 4 \\
\hline Discutir a Gamificação & 2 \\
\hline Engajar & 24 \\
\hline Maximizar o Aprendizado & 19 \\
\hline Mudança de Comportamento & 19 \\
\hline Socialização & 13 \\
\hline
\end{tabular}

Apenas um estudo cita a aprendizagem colaborativa apoiada por computador como um dos recursos disponíveis para o desenvolvimento de aplicações que enfatizam a socialização dos estudantes e a formação de grupos. Entretanto, nenhum dos estudos aborda diretamente a aprendizagem colaborativa apoiada por computador, o que responde a questão de pesquisa $\mathbf{Q P}_{\mathbf{3}}$. 


\section{Ameaças à Validade}

Para garantir um processo de seleção imparcial, as questões de pesquisa e os critérios de inclusão e exclusão foram criados antes do início do mapeamento. Os autores realizaram a seleção dos estudos de forma independente e ao final eventuais desacordos e dúvidas foram sanadas. Somente um conjunto limitado de base de dados foram utilizados, portanto é possível que estudos relevantes não tenham sido incluídos. A coerência do sistema de classificação elaborado pode significar uma ameaça a validade já que o conhecimento necessário para elaborá-lo, de acordo com Pretorius e Budgen (2008) muitas vezes só é obtido ao final da seleção.

\section{Considerações Finais}

O principal objetivo desse estudo de mapeamento é proporcionar uma visão geral do que tem sido investigado no contexto da gamificação aplicada à educação. Para cumprir esse objetivo, seguiu-se uma metodologia sistemática, ou seja, o mapeamento sistemático. Para tal foram definidas três questões de pesquisa a serem respondidas pelo mapeamento. $\mathbf{Q P} \mathbf{P}_{\mathbf{1}}$ : Em quais contextos e níveis educacionais gamificação tem sido mais investigada? $\mathbf{Q P}_{2}$ : Que tipos de estudos têm sido mais investigados em gamificação e educação? $\mathbf{Q P}$ : Que abordagens de gamificação tem sido mais investigada no campo da CSCL? De acordo com os resultados, a maioria dos estudos foram publicados em conferências e têm se concentrado sobre o Ensino Superior $\left(\mathbf{Q P}_{\mathbf{1}}\right.$ - ver Figura 1) para promover o envolvimento dos alunos através de atividades de aprendizagem que se baseiam em conceitos de gamificação $\left(\mathbf{Q P}_{2}\right.$ - ver Tabela 2). Também identificou-se que existe uma falta de abordagens que combinem gamificação e CSCL $\left(\mathbf{Q P}_{3}\right)$.

A novidade desta pesquisa é que, com o melhor do conhecimento dos autores, este é o primeiro mapeamento sistemático que abrange a investigação em gamificação aplicada à educação. Outra contribuição desta pesquisa é o mapa (Figura 4) criado. Ao analisar esse mapa é possível identificar de que forma a gamificação tem sido explorada e em que contextos educativos, revelando áreas até então pouco investigadas e futuras oportunidades de pesquisa.

\section{Agradecimentos}

Os autores agradecem o suporte financeiro provido pela Coordenação de Aperfeiçoamento de Pessoal de Nível Superior (CAPES) e pelo Conselho Nacional de Desenvolvimento Científico e Tecnológico - CNPq (Processos 400481/2013-8 e 476566/2011-8).

\section{Referências}

Domínguez, A., Saenz-de-Navarrete, J., de-Marcos, L., Fernández-Sanz, et al. (2013) Gamifying learning experiences: Practical implications and outcomes, Computers \& Education, 63(April), pages 380-392.

Gee, J. P. (2003) What video games have to teach about learning and literacy, Palgrave Macmillan, New York.

Kapp, K. M. (2012) The Gamification of learning and instruction: Game-based methods and strategies for training and education. Pfeiffer. Hoboken, NJ. 
Klopfer, E. (2008) Augmented learning: Research and design of mobile educational games. The MIT Press.

Lee, H., and Doh, Y. Y. (2012) A Study on the relationship between educational achievement and emotional engagement in a gameful interface for video lecture systems. International Symposium on Ubiquitous Virtual Reality, pages 34-37.

Lepper, M. R. and Cordova, D. I. (1996) Intrinsic motivation and the process of learning: Beneficial effects of contextualization, Pprsonalization, and choice. Journal of Educational Psychology, 88(4), pages 715-730.

Ma, M., Oikonomou, A. V., \& Jain, L. C. (2011) Serious games and edutainment applications. London: Springer-Verlag London Ltd.

Malone, T. W. and Lepper, M. R. (1987) Making learning fun: A Taxonomy of intrinsic motivations for learning. In Aptitude, Learning, and Instruction: Vol. 3. Lawrence Erlbaum, Hillsdale, NJ.

Petersen, K., Feldt, R., Mujtaba, S. and Mattsson, M. (2008) Systematic mapping studies in software engineering. In Proceedings of the international conference on Evaluation and Assessment in Software Engineering, 68-77.

Pretorius, R. e Budgen, D. (2008) A Mapping study on empirical evidence related to the models and forms used in the UML. In ACM-IEEE International Symposium on Empirical Software Engineering and Measurement, pages 342-344.

Shaffer, D. W. (2006) How computer games help children learn. Palgrave Macmillan, New York. 